\title{
Magnetic resonance imaging of coarctation of the aorta in infants: use of a high field strength
}

\author{
E J BAKER, VICTORIA AYTON, * M A SMITH, * J M PARSONS, M N MAISEY, * \\ E J LADUSANS, R H ANDERSON, $\dagger$ M TYNAN, A K YATES, $\ddagger$ P B DEVERALL $\ddagger$ \\ From the Departments of Paediatric Cardiology, ${ }^{\star}$ Radiological Sciences, and $\ddagger$ Cardiothoracic Surgery, Guy’s \\ Hospital, and $\uparrow$ National Heart and Lung Institute, London
}

SUMMARY Nineteen infants with suspected coarctation of the aorta were studied with electrocardiographically gated magnetic resonance imaging on a $1.5 \mathrm{~T}$ whole body imaging system. In all cases imaging was successful and produced diagnostic images of high resolution. Coarctation was shown in 12 cases. The position and shape of the coarctation were well displayed by the magnetic resonance images. In addition, they clearly showed the relation of the coarctation to arteries arising from the aortic arch and to the length and diameter of the aortic isthmus and the distal aortic arch. The anatomy was confirmed at operation in all 12 patients, except for two with a small ductus arteriosus (arterial duct), which was not seen in the magnetic resonance images. In the seven remaining patients, coarctation was excluded.

Magnetic resonance imaging produced high quality images that showed the anatomy better than other non-invasive methods. It provided all the anatomical information required for surgical correction.

Magnetic resonance has been promoted as an important advance in the imaging of congenital malformations of the heart and great vessels. ${ }^{1}$ As yet, however, there is little evidence that it will either supplant or supplement established imaging techniques. There is no experience in very young children, the age group in which most cases of coarctation now present. ${ }^{2}$ We report our experience of imaging the aorta in a group of infants with suspected coarctation with a high field strength magnetic resonance imaging system. The superior spatial resolution of such a system makes it particularly suitable for this age group.

\section{Patients and methods}

\section{PATIENTS}

Nineteen infants, aged between three days and 10 months, were imaged (table). In every case there was clinical or echocardiographic suspicion of coarctation of the aorta. Infants who were in severe heart failure that required intravenous inotropic support or ven-

Requests for reprints to Dr E J Baker, Department of Paediatric Cardiology, Guy's Hospital, London SE1 9RT.

Accepted for publication 11 April 1989 tilation were not included; otherwise there were no selection criteria. Four of the patients studied (patients 4, 5, 6, and 10) subsequently had aortograms. Surgical correction was performed in all the cases in which coarctation was confirmed by magnetic resonance imaging.

\section{METHODS}

The infants were fed just before the imaging procedure and, if necessary, sedated with $75 \mathrm{mg} / \mathrm{kg}$ of chloral hydrate. They were imaged by a $1.5 \mathrm{~T}$ whole body imaging system (Gyroscan) manufactured by Philips Medical Systems. Electrocardiogram electrodes were placed closely around the left nipple, an arrangement that minimises the distortion of the electrocardiogram produced by the movement of blood within the high magnetic field. The electrocardiogram was transmitted by a telemetry system. The infants were wrapped in a blanket and their movement was restricted by a firm foam mattress curved in a semicircle around them. They were imaged while supine in a $32 \mathrm{~cm}$ diameter proton head coil.

Electrocardiographically gated sections $(5 \mathrm{~mm}$ thick), separated by $0.5 \mathrm{~mm}$, were simultaneously acquired by a spin echo sequence with an echo time of 
Table Details of patients studied

\begin{tabular}{|c|c|c|c|}
\hline Number & Age & Anatomy shown by magnetic resonance & $\begin{array}{l}\text { Surgical } \\
\text { findings } \\
\text { where different }\end{array}$ \\
\hline $\begin{array}{r}1 \\
2 \\
3 \\
4 \\
5 \\
6 \\
7 \\
8 \\
9 \\
10 \\
11 \\
12 \\
13 \\
14 \\
15 \\
16 \\
17 \\
18 \\
19\end{array}$ & $\begin{array}{l}3 \text { wk } \\
3 \text { wk } \\
8 \text { days } \\
17 \text { days } \\
6 \text { days } \\
2 \text { mnth } \\
5 \text { days } \\
6 \text { wk } \\
10 \text { mnth } \\
3 \text { mnth } \\
3 \text { days } \\
3 \text { mnth } \\
1 \text { mnth } \\
2 \text { mnth } \\
10 \text { days } \\
2 \text { mnth } \\
12 \text { days } \\
7 \text { wk } \\
6 \text { wk }\end{array}$ & $\begin{array}{l}\text { Discrete shelf, mild isthmal hypoplasia } \\
\text { Waist lesion, no isthmus, mild distal arch hypoplasia } \\
\text { Discrete shelf, severe isthmal hypoplasia, patent arterial duct } \\
\text { Mild isthmal narrowing, no coarctation } \\
\text { Discrete shelf, anomalous right subclavian artery proximal to coarctation } \\
\text { Anomalous pulmonary veins to coronary sinus, coarctation excluded } \\
\text { No coarctation } \\
\text { Discrete shelf, mild isthmal hypoplasia, ventricular septal defect } \\
\text { Waist lesion, no isthmus } \\
\text { Hypoplasia of abdominal aorta, no coarctation } \\
\text { Normal aortic arch } \\
\text { Discrete shelf, mild isthmal hypoplasia } \\
\text { No isthmus, hypoplasia of distal arch, ventricular septal defect, subaortic infundibulum } \\
\text { Mild isthmal hypoplasia, discrete shelf, small ventricular septal defect } \\
\text { Mild isthmal hypoplasia, no coarctation } \\
\text { Severe isthmal hypoplasia, discrete shelf } \\
\text { Normal aortic arch } \\
\text { Waist lesion, hypoplasia of distal arch, no isthmus } \\
\text { Discrete shelf, distal arch and isthmal hypoplasia }\end{array}$ & $\begin{array}{l}\text { Small arterial duct } \\
\text { Agree } \\
\text { Agree } \\
\overline{\text { Agree }} \\
\overline{-} \\
\text { Agree } \\
\text { Agree } \\
\text { - } \\
\text { Agree } \\
\text { Small arterial duct } \\
\text { Agree } \\
\text { Agree } \\
\overline{\text { Agree }} \\
\text { Agree }\end{array}$ \\
\hline
\end{tabular}

$30 \mathrm{~ms}$. The matrix used was $256 \times 256$, with a typical pixel size of $0.75 \mathrm{~mm}$. To reduce acquisition time we used 180 phase encoding gradients instead of the usual 256 to produce the image. Two acquisitions of each phase encoding step were averaged. This resulted in a typical scan time for one set of seven sections of about six minutes.

In every case, the initial series of sections was in the transverse plane of the body, encompassing the structures between the arch of the aorta and the diaphragm. This, typically, required seven to nine sections. Subsequent imaging planes, oblique to the standard planes of the body, were chosen to show the area of suspected coarctation. The angles for these planes, chosen individually in each patient, were determined directly from the initial series of transverse images. The most valuable imaging plane for the aortic arch was found to be an oblique'sagittal, rotated around a vertical axis, to show the aortic arch (figs 1-5). Occasionally, the imaging plane had to be additionally rotated around an anterioposterior axis to show the aortic arch optimally. A coronal plane, which showed the descending aorta, was also useful in some cases.

In all the patients with coarctation, the area of narrowing was shown in the oblique sagittal plane and at least one other plane, either the transverse or coronal. In most patients it was imaged in all three planes.

\section{Results}

Images of diagnostic quality were obtained in every patient. There were no imaging failures. In all patients the electrocardiographic gating was satisfac-

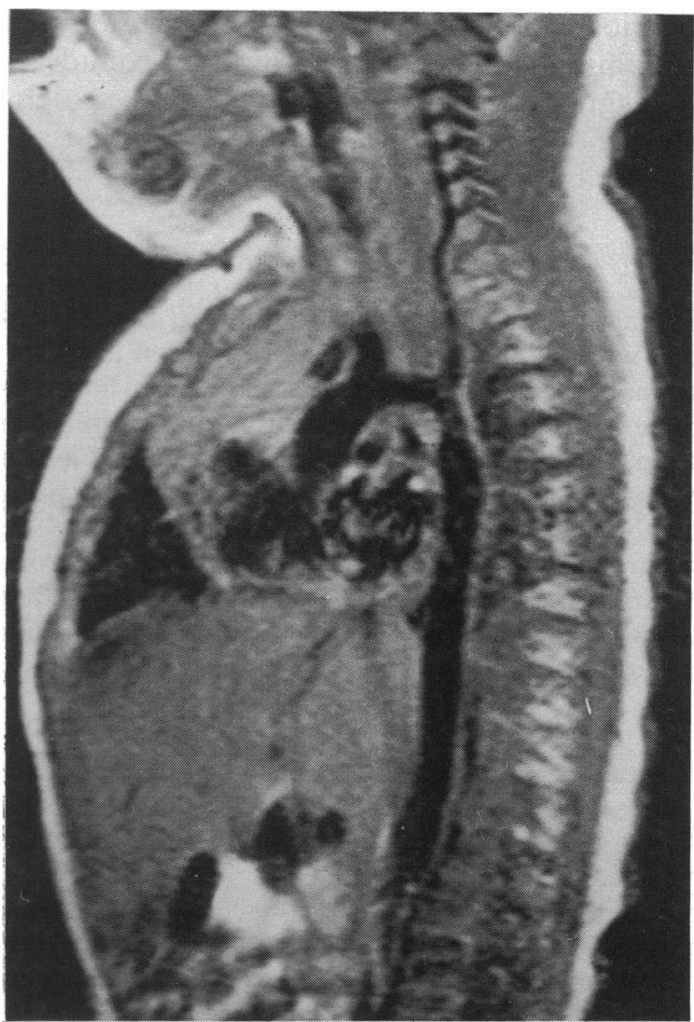

Fig 1 A normal aortic arch in a three day old infant (patient 11). This image is in an oblique sagittal plane rotated around a vertical axis. There is some mild isthmal narrowing but no coarctation. Cross sectional echocardiography did not rule out the presence of coarctation. 


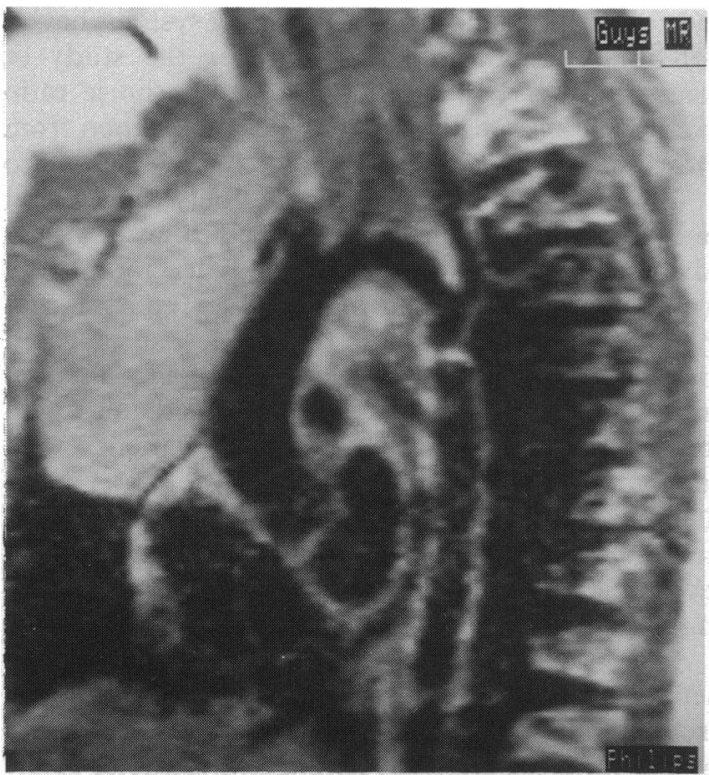

Fig 2 An oblique sagittal section in a three month old infant with coarctation. There is a discrete shelf across the descending aorta. The length of the aortic isthmus and the relation of the coarctation to the left subclavian artery is clearly seen. There is only very mild isthmal hypoplasia.

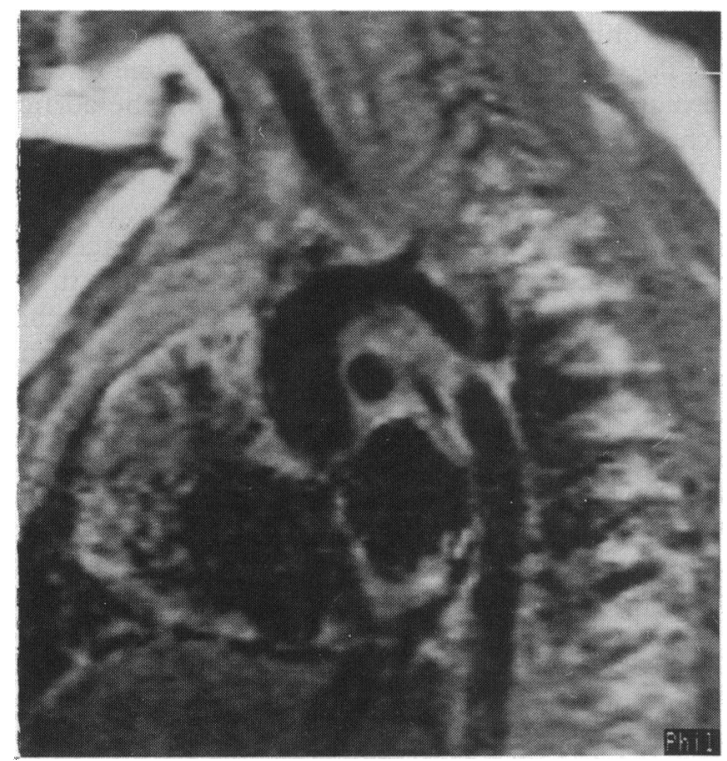

Fig 3 An oblique sagittal section in another patient aged 10 months with coarctation. In contrast with the anatomy in figure 2, there is a waist like narrowing of the descending aorta. The origin of the left subclavian artery arises at the level of the coarctation and so there is no isthmus.

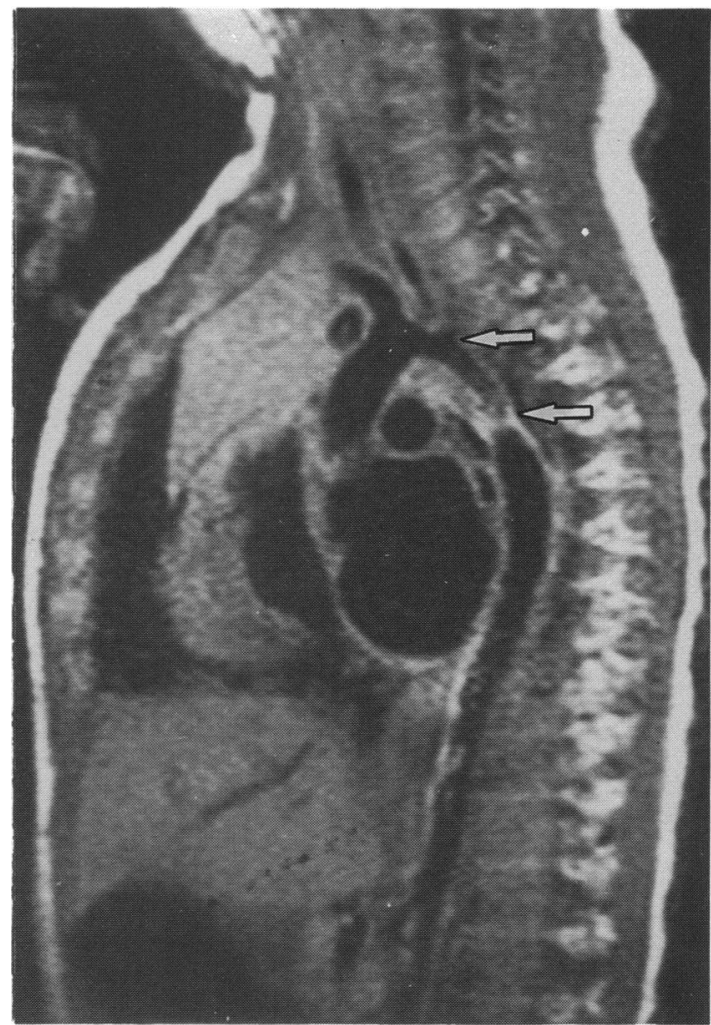

Fig 4 An oblique sagittal section in an infant with a very long, hypoplastic isthmus (between the arrows). The coarctation forms a discrete shelf rather than a waist.

tory. The diagnosis of coarctation was confirmed in 12 infants. It was excluded in seven. In the first case in which it was excluded (patient 4), we lacked experience in interpreting the magnetic resonance images and so the clinical picture prompted investigation by angiography. The aortogram confirmed normal aortic anatomy. In all the subsequent cases the diagnosis based on magnetic resonance imaging was accepted. Angiography was performed in two other negative cases (patients 6 and 10) to define aspects of the cardiac anatomy other than the aortic arch. In one positive case the arrangement of the branches of the aortic arch was unclear. An aortogram was performed before surgical correction to clarify this. The right subclavian artery arose anomalously just proximal to the coarctation and, in retrospect, this could be seen in the coronal images of the coarctation in this patient. In all the remaining positive cases, surgical correction was performed without further investigation.

Surgical correction confirmed the anatomy in all the patients with coarctation. But in two patients small arterial ducts (ductus arteriosus) were found at 


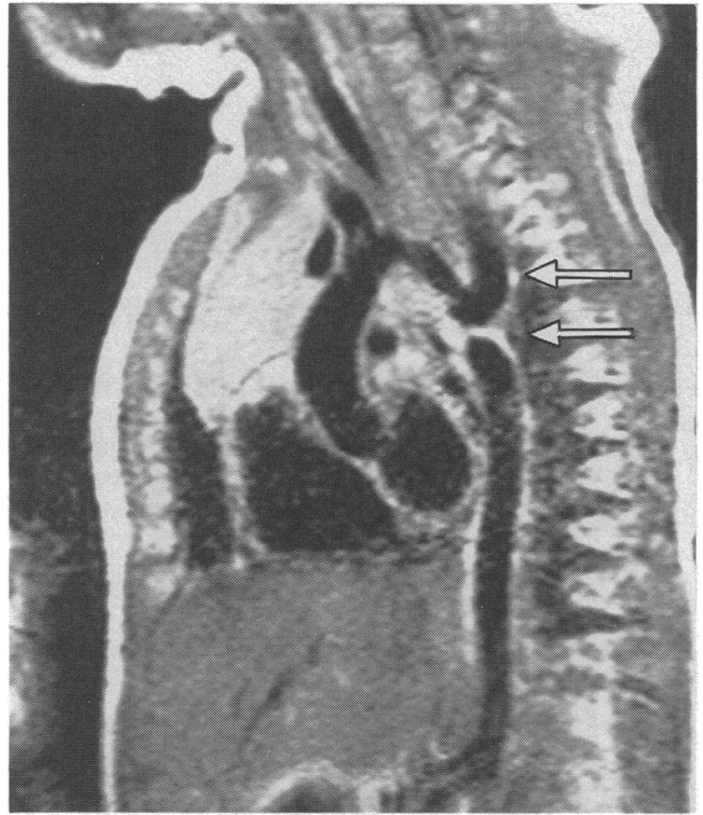

Fig 5 An oblique sagittal section in an infant with coarctation in the form of a shelf (lower arrow). There is no isthmus and a dilated left subclavian artery (upper arrow) can be seen arising just proximal to the shelf. In this case there is considerable hypoplasia of the distal aortic arch proximal to the origin of the left subclavian artery.

operation that were not seen in the magnetic resonance images. In practice this was not of clinical importance. In one patient a large arterial duct was shown by magnetic resonance imaging (case 3 ).

The presence or absence of obstruction in the thoracic aorta could invariably be shown in the initial transverse series of images. Further oblique sagittal images were, however, obtained in all cases to display the anatomy of the aortic arch clearly. From such images the diameter and length of the aortic isthmus were evident. The coarctation shelf, itself, gave a very intense magnetic resonance signal in these $\mathrm{T} 1$ weighted images. Images obtained in the coronal plane were valuable in defining the relation of the coarctation to the vessels arising from the aortic arch, particularly the left subclavian artery.

\section{Discussion}

To date magnetic resonance imaging of the heart has generally been performed with systems that use low and medium field strengths, $\leqslant 0.6 \mathrm{~T}^{34}$ It was thought that it would be difficult to achieve cardiac gating in strong magnetic fields such as $1.5 \mathrm{~T}$. This is because the electrocardiographic signal is distorted by the flow of blood in the high magnetic field. But the newer, high field strength imaging systems have a potentially important advantage for the study of congenital heart disease. The signal to noise ratio from these systems is considerably better than from those that use low and medium strength fields and so a more favourable compromise between spatial resolution and imaging time can be achieved. These advantages are especially valuable when infants are studied. A major criticism of published reports is that there is almost no experience in the use of gated magnetic resonance cardiac imaging in infants. Indeed, most workers specifically avoided imaging in this age group. Because almost all patients with congenital heart disease present in the first year of life, ${ }^{2}$ the anatomical diagnosis of cardiac defects is now generally performed in infancy. An imaging technique, to be of value, must be capable of producing high quality images in this age group.

As we have shown, magnetic resonance images can define the position of the coarctation in the aortic arch, its relation to the arterial duct and the arteries arising from the arch, the length and diameter of the aortic isthmus, and whether the coarctation is a discrete shelf or a waist lesion. ${ }^{5}$ Identification of the coarctation is greatly helped by the intensity of the signal from the shelf itself. In infants, this is usually made up of tissue originating from the arterial duct (ductus arteriosus). ${ }^{6}$ We always made certain that the shelf could be seen in images in at least two different planes, thus ensuring it was not an artefact produced by the oblique transection of the aorta. In practice, this does not seem to be a problem. In none of our normal patients were the appearances of the aortic arch ambiguous.

We believe that our experience shows that high field strength magnetic resonance imaging can consistently produce high resolution cardiac images in infants. In this series, we have shown that it is possible, with great clarity, to image the aortic arch. The images were of much higher resolution than those obtained with cross sectional echocardiography. ${ }^{7}$ In addition, magnetic resonance imaging is non-invasive and safe. It does not use any ionising radiation. It provides all the anatomical information required for surgical repair of the anomaly. It thus has many advantages for studying this age group. Its clinical role requires further evaluation.

This research was supported by the British Heart Foundation. The imaging system was purchased with the assistance of Sir Philip and Lady Harris and the Special Trustees of Guy's Hospital.

\section{References}

1 Didier D, Higgins CB, Fischer MR, Osaki L, Silverman 
NH, Cheitlin MD. Congenital heart disease: gated MR imaging in 72 patients. Radiology 1986;158: 227-35.

2 Hoffman JIE, Christianson R. Congenital heart disease in a cohort of 19,502 births with long term follow up. Am J Cardiol 1978;42:641-7.

3 Boxer RA, LaCorte M, Singh S, et al. Nuclear magnetic resonance imaging in evaluation and follow-up of children treated for coarctation of the aorta. J Am Coll Cardiol 1986;7:1095-8.

4 von Shulthess GK, Higashino SM, Higgins SS, Didier D, Fischer MR, Higgins CB. Coarctation of the aorta:
MR imaging. Radiology 1986;158:289-96.

5 Sinha SN, Kardatzke ML, Cole RB, Muster AJ, Wessel HU, Paul MH. Coarctation of the aorta in infancy. Circulation 1969;40:385-98.

6 Elzenga NJ, Gittenberger-de Groot AC, OppenheimerDekker A. Coarctation and other obstructive aortic arch anomalies: their relationship to the ductus arteriosus. Int J Cardiol 1986;13:289-308.

7 Smallhorn JF, Huhta JC, Adams PA, Anderson RH, Wilkinson JL, Macartney FJ. Cross-sectional echocardiographic assessment of coarctation in the sick neonate and infant. Br Heart J 1983;50:349-61. 